\title{
The role of low-density lipoprotein (LDL) and high-density lipoprotein (HDL) in comparison with whole egg yolk for sperm cryopreservation in rhesus monkeys
}

\author{
Qiao-Xiang Dong ${ }^{1,2}$, Sarah E Rodenburg ${ }^{2}$, Dana Hill ${ }^{2}$ and Catherine A VandeVoort ${ }^{2,3}$
}

Low-density lipoprotein (LDL) extracted from hen egg yolk has recently been considered to be superior to whole egg yolk in sperm cryopreservation of various animal species. Meanwhile, there was a notion that high-density lipoprotein (HDL) in egg yolk may have a negative effect on post-thaw survival. The role of LDL and HDL in sperm cryopreservation of rhesus monkeys has not been explored. The present study evaluates their effect in comparison with egg yolk with or without the addition of permeable cryoprotectant (glycerol) on sperm cryopreservation of rhesus macaques. In addition, various additives intended to change the lipid composition of LDL-sperm membrane complex have also been tested for their effectiveness in preserving post-thaw viability. Our findings indicated that LDL is the main component in egg yolk that is responsible for its protective role for sperm cryopreservation in rhesus monkeys. Regardless of the presence or absence of glycerol, the protective role of LDL is similar to that of egg yolk and we did not observe any superiority in post-thaw survival with LDL when compared to egg yolk. Modifying the lipid composition of LDL-sperm membrane complex with the addition of cholesterol, cholesterol loaded cyclodextrin and phosphatidylcholine also did not yield any improvements in post-thaw survival; while addition of methyl- $\beta$-cyclodextrin reduced post-thaw motility. HDL plays a neutral role in sperm cryopreservation of rhesus monkeys. The present study suggests that egg yolk may still hold advantages when compared with LDL as effective components in extenders for sperm cryopreservation in rhesus monkeys. Asian Journal of Andrology (2011) 13, 459-464; doi:10.1038/aja.2010.145; published online 21 March 2011

Keywords: non-human primates; sperm cryopreservation; low-density lipoprotein; high-density lipoprotein; egg yolk

\section{INTRODUCTION}

Egg yolk constitutes one of the most important ingredients in routine freezing extenders for sperm cryopreservation of various animal species. However, the potential confounding of experiments from the variability in yolk composition from different egg sources has driven the need to find defined replacements. Though the underlying mechanism of how egg yolk helps sperm survive the cryopreservation process is unknown, it is generally believed that the low-density lipoprotein (LDL) is the main component that offers protection. ${ }^{1-4}$ Recent advances in methods for simple extraction of LDL by Moussa et $a l^{5}$ promoted a series of studies to test the effectiveness of using LDL to replace whole egg yolk for sperm cryopreservation (Table 1). A general trend of superiority in using 6\%-9\% LDL instead of $20 \%$ egg yolk was demonstrated in dog, bull, boar and sheep, but not in the Iberian red deer (Table 1).

Hen egg yolk accounts for $36 \%$ of the weight of the whole fresh egg. ${ }^{6}$ On the basis of its dry matter, yolk is constituted of $68 \%$ LDL, $16 \%$ high-density lipoprotein (HDL), 10\% globular proteins (livetines), $4 \%$ phosphoproteins (phosvitin) and $2 \%$ minor proteins. ${ }^{7}$ Since LDL is thought to be the main component responsible for the protective role of egg yolk in sperm cryopreservation, many groups have advocated its replacement for whole egg yolk, because of concerns associated with egg yolk. For example, yolk granules (which include
HDL) were suspected to have negative effects on sperm viability. ${ }^{1,8}$ The presence of progesterone in egg yolk has also been considered to counteract cryopreservation success due to its inducible role in sperm capacitation. ${ }^{9}$ In addition, some egg yolk components are known to interfere with laboratory biochemical assays and metabolic parameters. ${ }^{10}$ In fact, the majority of recent studies indicate that the improvement in post-thaw viability with LDL is due to the removal of these negative effects in the whole egg yolk (Table 1).

The role of LDL and HDL in sperm cryopreservation of non-human primates has not been explored. To evaluate whether LDL will exhibit similar advantages while HDL will exhibit detrimental effects in postthaw survival for monkey sperm, we studied the effect of LDL and HDL with or without addition of permeable cryoprotectant (glycerol) in comparison with egg yolk on sperm cryopreservation of rhesus macaques. In addition, various additives intended to change the lipid composition of LDL-sperm membrane complex have also been tested for their effectiveness in preserving post-thaw viability.

\section{MATERIALS AND METHODS}

Semen collection and processing

Rhesus monkeys (Macaca mulatta) were housed at the California National Primate Research Center. All procedures for maintenance and handling of the animals were reviewed and approved in advance 
Table 1 Post-thaw viability (\%) of sperm samples frozen with $20 \%$ EY versus LDL in various animals

\begin{tabular}{|c|c|c|c|c|c|c|c|c|c|c|c|c|}
\hline Species & Dog & Dog & Dog & Bull & Bull & Bull & Bull & Boar & Boar & Agu pig & $\begin{array}{l}\text { Iberian } \\
\text { red deer }\end{array}$ & Sheep \\
\hline \% LDL range & $6-10$ & $4-10$ & 6 & $7-10$ & 8 & 8 & $2.5-20$ & 9 & $6-10$ & $2-10$ & 8 & $5-15$ \\
\hline Optimal \% LDL & 8 & 6 & 6 & 8 & 8 & 8 & 8 & 9 & 9 & $4-6$ & 8 & 9 \\
\hline MI-HOS (PI stain) & $20 / 42$ & $46 / 56$ & $65 / 66$ & $42 / 52$ & $47 / 53$ & $46 / 47$ & - & $31 / 51$ & $44 / 53$ & $E Y<L D L$ & $52 / 40$ & $30 / 35$ \\
\hline Al-PSA (PNA) [Gm] & - & $57 / 81$ & $53 / 55$ & $43 / 70$ & - & 92/92 & - & $37 / 71$ & $42 / 50$ & - & $78 / 73$ & $55 / 65$ \\
\hline Mito-Rh123 (JC) [MTR] & - & - & - & $42 / 53$ & - & - & - & - & $68 / 79$ & $E Y<L D L$ & $42 / 58$ & - \\
\hline IVF-blastula & - & - & - & - & - & $24 / 22$ & - & - & - & - & - & - \\
\hline Reference & 39 & 9 & 37 & 40 & 41 & 42 & 5 & 43 & 44,45 & 10 & 46 & 47 \\
\hline
\end{tabular}

Abbreviations: EY, egg yolk; LDL, Low-density lipoprotein; Mot-CASA, motility (measured as computer assisted sperm analysis or subjectively); MI-HOS (PI stain), membrane integrity (hypoosmotic swelling or propidium iodide staining); AI-PSA (PNA) [Gm], acrosome integrity (measured as peptide nucleic acid-FITC or peanut agglutinin-FITC or Giemsa staining); Mito-Rh123 (JC) [MTR], mitochondrial activity (Rhodamine 123 probe; miochondrial probe JC-1: 5,5',6,6'-tetrachloro-1,1',3,3'-

tetraethylbenzimidazolocarbocyanine iodide; MitoTracker Red CMXRos); DNA-AO (comet), DNA integrity (measured using acridine orange or comet assay); IVF, in vitro fertilization (cleavage rate, penetrating rate or blastula rate); -, data not available.

by the Institutional Animal Use and Care Administrative Advisory Committee at the University of California at Davis. Semen samples were collected from eight adult males that were individually caged at the California National Primate Research Center with lights on from 06:00 to $18: 00$ h at $25-27^{\circ} \mathrm{C}$. The males were trained to chair restraint and semen was collected by direct penile stimulation with a Grass 6 stimulator equipped with EKG pad electrodes (30-50 V, 20-ms duration, 18 pulses $\left.\mathrm{s}^{-1}\right){ }^{11}$ Samples were allowed to liquefy for $30 \mathrm{~min}$ before processing. Sperm suspensions were washed twice with a modified Tyrode's medium supplemented with $3 \mathrm{mg} \mathrm{ml}^{-1}$ bovine serum

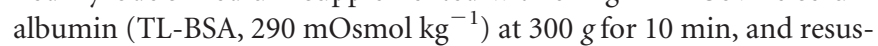
pended to $2 \times 10^{8}$ cells ml $^{-1}$ of total motile sperm (sperm density $\times$ initial motility) with TEST solution (43.25 g TES, $10.265 \mathrm{~g}$ Tris, $10 \mathrm{~g}$ glucose in one liter distilled water, $\mathrm{pH} 7.4,350 \mathrm{mOsmol} \mathrm{kg}{ }^{-1}$ ) before subject to various treatments. Sperm were frozen at a final concentration of $5 \times 10^{7}$ cells $\mathrm{ml}^{-1}$. Unless specified otherwise, all chemicals used for preparation of solutions were of reagent grade (Sigma Chemical Corporation, St Louis, MO, USA). A total of 46 ejaculates were used in this study. Among which data from 40 ejaculates that subjected to the same control treatment ( $20 \%$ egg yolk alone without glycerol) were analyzed to examine the male variation, and no male effect was found among these males $(P>0.05$, Figure 1$)$.

\section{Freezing and thawing procedure}

Aliquots $(50 \mu \mathrm{l})$ of sperm suspensions were drawn into 0.25 -ml French straws (IMV International, Minneapolis, MN, USA) manually with a 1cc syringe and were heat sealed (MP-4 Impulse Sealer, Midwest Pacific; JJ Elemer Corporation, St. Louis, MO, USA). Straws were placed into a 600$\mathrm{ml}$ glass beaker containing $500 \mathrm{ml}$ of room temperature distilled water, and equilibrated at $4{ }^{\circ} \mathrm{C}$ in a refrigerator for $2 \mathrm{~h}$ before initiation of the freezing process. Freezing followed the methods described previously. ${ }^{12}$ In brief, a styrofoam box was filled with a depth of $4 \mathrm{~cm}$ liquid nitrogen and a 1-cm thick styrofoam 'boat' was floated on top of it for $10 \mathrm{~min}$, then straws were placed on top of the 'boat' for $10 \mathrm{~min}$ before being plunged into liquid nitrogen. For post-thaw motility estimation, three straws per treatment were thawed in a $37^{\circ} \mathrm{C}$ water bath for $30 \mathrm{~s}$. Samples were evaluated after a minimum of 1-week storage in liquid nitrogen.

\section{Motility estimation}

A $10-\mu \mathrm{l}$ drop of prefreeze or post-thaw semen, covered with a $22-\mathrm{mm}^{2}$ coverglass, was visualized with $\times 20$ positive-phase objective and a condenser setting of 100 (pseudodark field) on an Olympus BH-series phase-contrast microscope (Scientific Instrument Co., Sunnyvale, CA, USA) with a heated stage at $37^{\circ} \mathrm{C}$. The average initial motility was $92 \% \pm 6 \%$. Post-thaw motility was estimated after incubation at $37^{\circ} \mathrm{C}$ in $5 \% \mathrm{CO}_{2}$ for $1 \mathrm{~h}$. Forward progression was estimated with an adjusted motility index (AMI) as described previously. ${ }^{12}$ In brief, the percent forward progression was subjectively estimated with a five point scale, and this was integrated with the percent motility into one number with the formula as follows: $\mathrm{AMI}=($ scale value $/ 4) \times$ percent motility. Samples were presented in random order each time so that the operator did not know their identity.

We did not include other evaluation criteria such as sperm morphology, membrane or acrosome integrity in this study because post-thaw motility was the most sensitive indicator of sperm function in nonhuman primates. Previous studies with cynomolgus macaques have shown that motility was the most convincing parameter for sperm function analysis when compared with sperm acrosome integrity (evaluated with FITC-coupled peanut agglutinin). ${ }^{13}$ Our own observation with

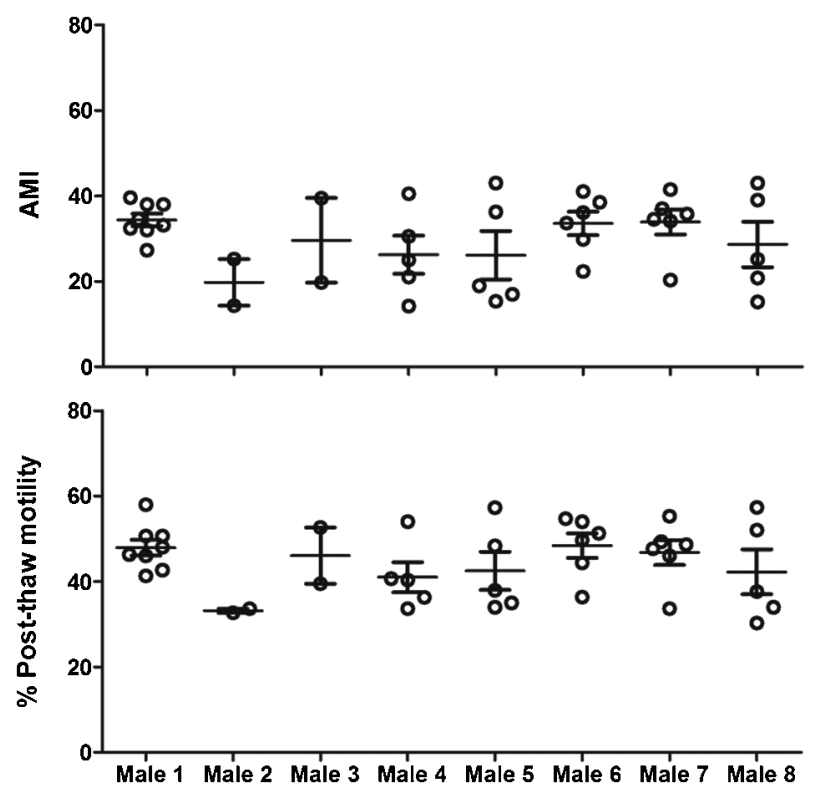

Figure 1 Post-thaw motility and AMI of 40 ejaculates derived from eight adult males of rhesus monkey that subjected to the control treatment of $20 \%$ egg yolk without glycerol. AMI, adjusted motility index. 
rhesus macaque also showed normal sperm head morphology, intact membrane and intact acrosome in many non-motile sperm after thawing.

\section{Extraction of LDL and HDL}

Detailed protocols for LDL and HDL extraction from egg yolk were provided by Dr M. Anton's laboratory at UR1268 Biopolymères Interactions Assemblages, Equipe Interfaces et Systèmes Dispersés, INRA, 44360 Nantes Cedex 3, France. In brief, yolk were separated out from egg white and washed with isotonic saline solution. Then yolk plasma (for LDL extraction) and granules (for HDL extraction) were separated with centrifugation at $10000 \mathrm{~g}$ for $45 \mathrm{~min}$ at $10^{\circ} \mathrm{C}$. For yolk plasma, ammonium sulfate ( $40 \%$ saturation) was added to precipitate livetins, which was discarded by centrifugation. The resulting supernatant was dialyzed against distilled water for $20 \mathrm{~h}$ at $4{ }^{\circ} \mathrm{C}$ with frequent water changes to remove ammonium sulfate. LDL aggregated after dialysis and was harvested by centrifugation at $10000 \mathrm{~g}$ for $45 \mathrm{~min}$ at $4{ }^{\circ} \mathrm{C} .{ }^{5}$ For $\mathrm{HDL}$ extraction, granules were rinsed with $\mathrm{NaCl}$ $0.17 \mathrm{~m} \mathrm{~mol}^{-1}$ to eliminate residue of plasma, and diluted with $\mathrm{NaCl}$ solution $1.74 \mathrm{~m} \mathrm{~mol}^{-1}$ to $10 \%(\mathrm{~m} / \mathrm{v})$. The solution was stirred overnight, the $\mathrm{pH}$ was adjusted to 7.25 , and the solution was dialyzed for $24 \mathrm{~h}$ in distilled water with frequent water changes to remove $\mathrm{NaCl}$ solution. The resulting pellets after centrifugation at $10000 \mathrm{~g}$ for $30 \mathrm{~min}$ at $4{ }^{\circ} \mathrm{C}$ are considered to be the HDL-rich fraction. ${ }^{14}$ The same batch of LDL and HDL were used throughout all experiments.

\section{Evaluation of LDL concentration}

Our previous studies have showed that rhesus monkey sperm can be effectively cryopreserved in $20 \%$ egg yolk alone without glycerol. ${ }^{15}$ To test whether LDL alone without permeable cryoprotectant yields similar post-thaw survival as that of egg yolk, sperm samples were first frozen with $20 \%$ egg yolk or $10 \%, 20 \%$ and 30\% LDL from eight ejaculates. Then in the second trial, five ejaculates were used to test the LDL at a lower concentration range of $2 \%, 4 \%, 6 \%, 8 \%$ and $10 \%$ including $20 \%$ egg yolk as a control.

\section{Evaluation of $10 \%$ LDL with various additives}

Lipid modification has been suggested to affect sperm tolerance to cryodamages. To evaluate whether changing the lipid composition of LDL would affect post-thaw survival, various additives were added to $10 \%$ LDL for freezing trials without permeable cryoprotectant. In the first trial, $1.6 \mathrm{mg} \mathrm{ml}^{-1}$ cholesterol-loaded cyclodextrin (CLC), $1.6 \mathrm{mg} \mathrm{ml}^{-1}$ cholesterol, $3 \mathrm{mg} \mathrm{ml}^{-1} \mathrm{~L}$ - $\alpha$-phosphatidylcholine (hen egg; Avanti Polar Lipids, Inc. Alabaster, AL, USA), and $20 \mathrm{~m} \mathrm{~mol}^{-1}$ methyl- $\beta$-cyclodextrin (MBCD) in combination with $10 \% \mathrm{LDL}$ were tested with eight ejaculates. In the second trial, 5 and $10 \mathrm{mg} \mathrm{ml}^{-1}$ of cholesterol and L- $\alpha$-phosphatidylcholine in combination with $10 \%$ LDL were tested with six ejaculates. Twenty percent of egg yolk and 10\% LDL were also included as controls.

\section{Evaluation of LDL in combination with glycerol}

To test whether LDL in combination with glycerol would yield similar post-thaw survival as that of egg yolk with glycerol, samples from six ejaculates were frozen in $20 \%$ egg yolk or $8 \%$ and $10 \%$ LDL with or without $3 \%$ glycerol (final concentration). In the second trial, samples from seven ejaculates were frozen in $2 \%, 4 \%, 6 \%, 8 \%$ and $10 \%$ LDL with $3 \%$ glycerol.

\section{Evaluation of HDL with and without glycerol}

To evaluate whether addition of HDL will have any adverse effect on post-thaw survival, sperm samples from six ejaculates were frozen in $20 \%$ egg yolk, TEST, $1 \%, 2 \%$ and $4 \%$ HDL with or without $3 \%$ glycerol.

\section{Data analysis}

Data were analyzed using one-way ANOVA (SAS 9.1, SAS Institute Inc., Cary, NC, USA). When a significant difference $(P<0.05)$ was observed among treatments, Tukey's studentized range test was used for post-test comparisons. Percent motility was arcsine-square root transformed and means of three straws per treatment were used for analysis. Values presented are means \pm s.d.

\section{RESULTS}

\section{Evaluation of LDL concentration}

When samples were frozen without permeable cryoprotectant, LDL in a concentration range of $10 \%-30 \%$ yielded similar post-thaw motility $(33 \%-40 \%)$ and AMI (17-24) as that of samples frozen in $20 \%$ egg yolk alone (39\% $\pm 7 \%$ for motility and $24 \pm 9$ for AMI). However, LDL at concentrations lower than $8 \%$ reduced post-thaw motility and AMI when compared with controls at $20 \%$ egg yolk $(P<0.05$; Figure 2$)$.

\section{Evaluation of $10 \%$ LDL with various additives}

The addition of cyclodextrin-loaded cholesterol, cholesterol and L- $\alpha$ phosphatidylcholine did not yield any beneficial effects on post-thaw motility when compared with controls of $10 \%$ LDL or $20 \%$ egg yolk (Figure 3). On the contrary, the addition of $20 \mathrm{mmol} \mathrm{l}^{-1}$ methyl- $\beta$ cyclodextrin reduced post-thaw motility and AMI $(P<0.001$; Figure 3). Further testing with higher concentrations up to $10 \mathrm{mg} \mathrm{ml}^{-1}$ of cholesterol and L- $\alpha$-phosphatidylcholine did not yield any improvements in post-thaw survival $(P>0.05)$, neither did they cause any detrimental effects (data not shown).

\section{Evaluation of LDL in combination with glycerol}

Post-thaw motility of samples frozen in the presence of glycerol suspended in $8 \% \mathrm{LDL}(61 \% \pm 6 \%)$ or $10 \% \mathrm{LDL}(62 \% \pm 6 \%)$ was similar to that of $20 \%$ egg yolk $(60 \% \pm 7 \%)$. These three treatment groups also yielded a similar AMI of $44 \%$ (Figure 4). Again, there were no differences in post-thaw motility and AMI of samples frozen in $8 \% \mathrm{LDL}$, $10 \%$ LDL and $20 \%$ egg yolk without addition of glycerol $(P>0.05)$, though treatments without glycerol had lower post-thaw survival than

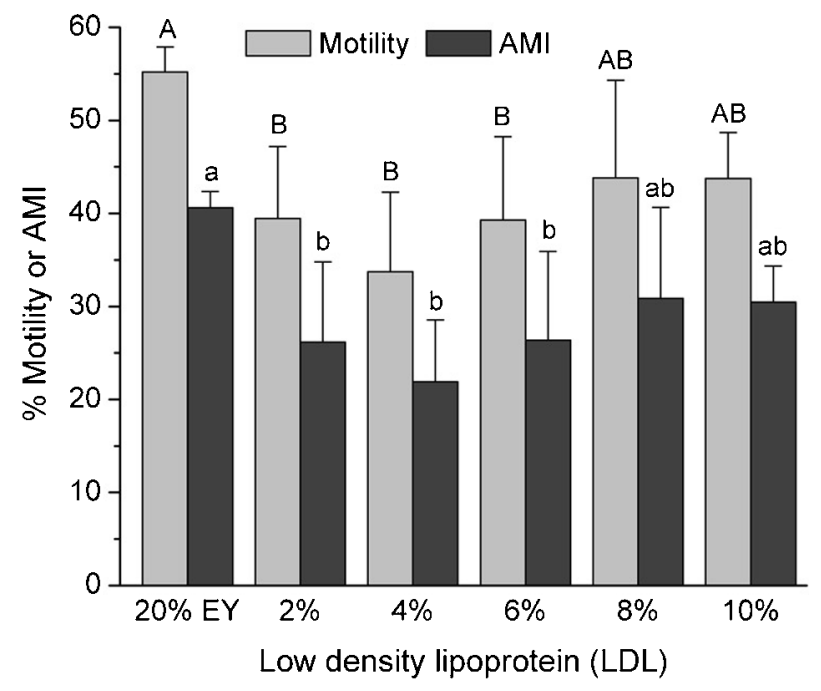

Figure 2 Post-thaw motility and AMI of rhesus monkey sperm samples frozen in $20 \%$ EY or $2 \%, 4 \%, 6 \%, 8 \%$ and $10 \%$ LDL without cryoprotectant. Bars with the different letter in upper case (for molity) or lower case (for $\mathrm{AMI}$ ) indicate significant difference. $P<0.05$. AMI, adjusted motility index; EY, egg yolk; LDL, lowdensity lipoprotein. 


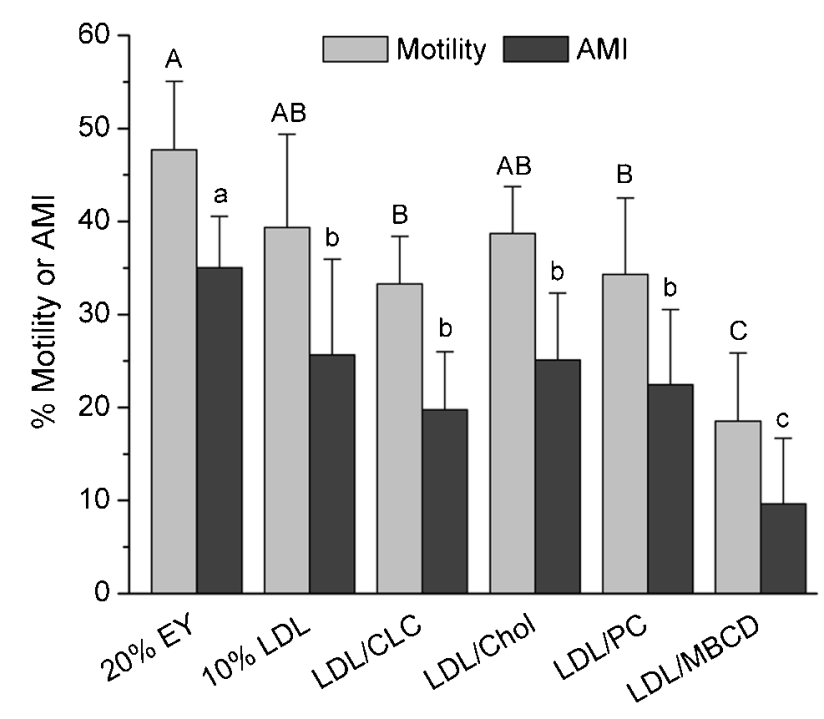

Figure 3 Post-thaw motility and AMI of rhesus monkey sperm samples frozen in $20 \% \mathrm{EY}, 10 \% \mathrm{LDL}$, or $10 \% \mathrm{LDL}$ with the addition of $1.6 \mathrm{mg} \mathrm{ml}^{-1}$ cyclodextrin loaded cholesterol (LDL/CLC), $1.6 \mathrm{mg} \mathrm{ml}^{-1}$ cholesterol (LDL/Chol), $3 \mathrm{mg} \mathrm{ml}^{-1} \mathrm{~L}$ $\alpha$-phosphatidylcholine (LDL/PC) and $20 \mathrm{~m} \mathrm{~mol} \mathrm{I}^{-1}$ methyl- $\beta$-cyclodextrin (LDL/ MBCD) without cryoprotectant. Bars with the different letter in upper case (for molity) or lower case (for AMI) indicate significant difference. $P<0.05$. AMI, adjusted motility index; EY, egg yolk; LDL, low-density lipoprotein.

those with glycerol ( $P<0.05$; Figure 4). With the addition of glycerol, LDL at lower concentrations $(2 \%-6 \%)$ yielded similar post-thaw motility (46\%-54\%) and AMI (28-34) as that of $8 \%$ LDL (53\% $0 \%$ for motility and $33 \pm 8$ for AMI) or $10 \%$ LDL ( $53 \% \pm 9 \%$ for motility and $36 \pm 10$ for AMI).

\section{Evaluation of HDL with and without glycerol}

When compared to TEST controls, the addition of 1\%-4\% HDL did not cause any detrimental effects on post-thaw motility or AMI in the presence or absence of glycerol (Figure 5). However, samples frozen in

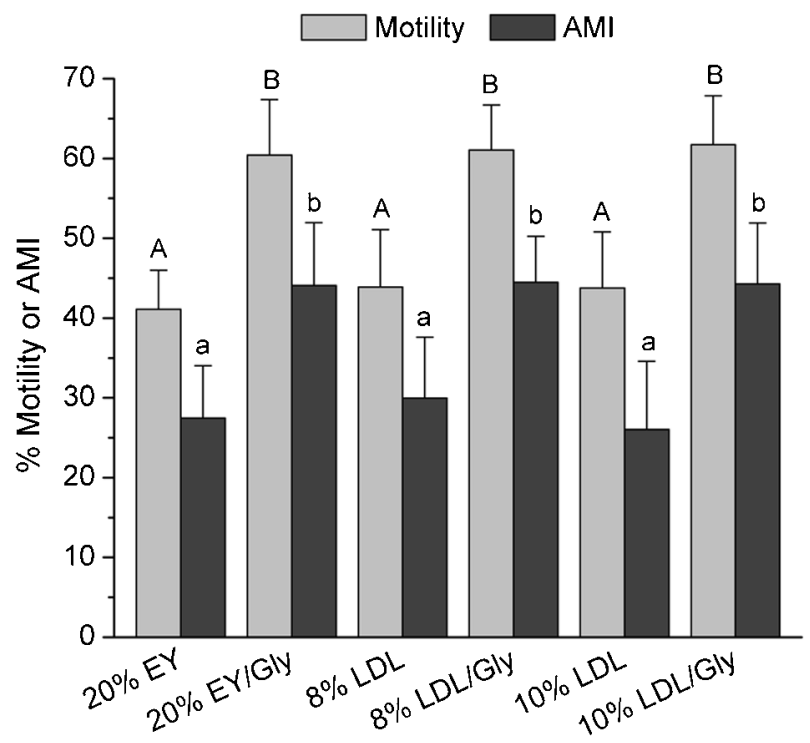

Figure 4 Post-thaw motility and AMI of rhesus monkey sperm samples frozen in $20 \%$ EY or 8 and 10\% LDL with or without 3\% Gly. Bars with the different letter in upper case (for molity) or lower case (for AMI) indicate significant difference. $P<0.05$. AMI, adjusted motility index; EY, egg yolk; LDL, low-density lipoprotein.
TEST or HDL yielded lower post-thaw motility and AMI than controls of $20 \%$ egg yolk both with and without the addition of glycerol $(P<0.05$; Figure 5).

\section{DISCUSSION}

In the present study, LDL did not offer any additional benefits in terms of post-thaw motility in comparison with $20 \%$ egg yolk regardless of the presence/absence of permeable cryoprotectant or addition of cholesterol or phosphatidylcholine. This finding did not agree with earlier studies on the superiority of LDL over egg yolk found in other species (Table 1). However, LDL in a concentration range of $6 \%-10 \%$ did yield similar post-thaw motility to that of $20 \%$ egg yolk in the presence of glycerol, which confirmed that LDL is the main component responsible for the protective role of egg yolk for sperm cryopreservation in rhesus macaques as $20 \%$ egg yolk contains $\sim 6 \%-7 \%$ LDL. $^{5}$ However, HDL did not cause any detrimental effects on post-thaw motility when compared with solution controls of TEST though neither did HDL contribute to any beneficial effects for post-thaw survival.

Egg yolk alone in the absence of permeable cryoprotectant has recently been demonstrated to protect sperm from the damage that occurs during the freeze-thaw process in rhesus monkeys. ${ }^{15}$ Similarly, the present study also showed similar effectiveness of LDL alone without glycerol in preserving monkey sperm as that of egg yolk. However, the effective LDL concentration varied with the presence of a permeable cryoprotectant. In the presence of glycerol, a wide range of LDL (2\%-20\%) yielded similar post-thaw survival compared to $20 \%$ egg yolk, while higher LDL concentrations ( $\geqslant 8 \%$ ) were necessary to obtain equal post-thaw survival as that of egg yolk in the absence of glycerol. Either LDL or glycerol alone exhibited lower post-thaw motility than these two combined, which suggests that LDL and glycerol act additively and perhaps through separate mechanisms to provide protection. This outcome agrees with our previous findings on the relationship between egg yolk and glycerol. ${ }^{15}$ Our observation of the wide tolerance of LDL concentrations $(2 \%-20 \%)$ in rhesus macaques further suggested that monkey sperm are more resistant to cryodamage than other animals such as bull, dog and boar (Table 1). A broad tolerance of macaque sperm to conditions required for cryopreservation has also been noted in studies of other sperm parameters such as osmotic stress, water permeability of membranes at room and freezing
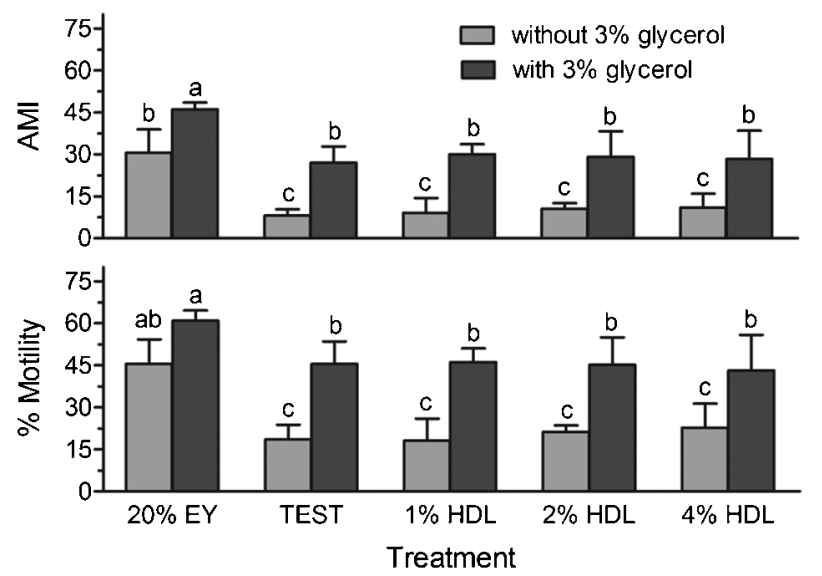

Figure 5 Post-thaw motility and AMI of rhesus monkey sperm samples frozen in $20 \%$ EY, TEST, or HDL at 1, 2 and $4 \%$ with or without $3 \%$ glycerol. Bars with the different letter indicate significant difference. $P<0.05$. AMI, adjusted motility index; EY, egg yolk; LDL, low-density lipoprotein. 
temperatures, and addition of cryoprotectants. ${ }^{16-18}$ Differences in optimal LDL concentrations for various species could be due to their difference in composition of the sperm plasma membranes. ${ }^{9}$

Because LDL consists of $86 \%-89 \%$ lipids and $12.5 \%$ proteins, ${ }^{19}$ lipids have been the main focus for the study of the protective role of egg yolk. ${ }^{20,21}$ One major source of injury during cryopreservation is membrane destabilization that occurs as temperature decreases and the fluid phase transitions into the gel phase. ${ }^{22}$ Some of this destabilization and damage can be prevented by the addition of lipids (reviewed by Ref. 23). Cholesterol is a major lipid component of mammalian sperm membranes, and rhesus sperm are no exception. ${ }^{24}$ High membrane cholesterol levels inhibit the crystallization of membrane hydrocarbon chains at low temperatures, thereby eliminating the phase transition. ${ }^{20}$ Increasing the cholesterol content of sperm membranes with CLC has been showed to improve cryosurvival of bull sperm, probably due to benefits of eliminating the phase transition and preventing premature sperm capacitation. ${ }^{20}$ On the other hand, decreasing the cholesterol to phospholipid $(\mathrm{C} / \mathrm{P})$ ratio can increase fluidity and permeability of animal plasma membranes. ${ }^{25}$ MBCD is a cyclic heptasaccharide that can eliminate much of the cholesterol from membranes and has been shown to improve cryosurvival of boar sperm. ${ }^{26}$ Increasing the phospholipid content of membranes is another way to alter the $\mathrm{C} / \mathrm{P}$ ratio and has been shown to improve cryosurvival of bull sperm. ${ }^{27}$

Much of this controversy may stem from variations in the initial status of $\mathrm{C} / \mathrm{P}$ ratio among different species or individuals. Thus, modification of LDL or sperm membrane lipids by adding or removing cholesterol or altering the $\mathrm{C} / \mathrm{P}$ ratio may improve the post-thaw sperm quality. In the present study, addition of cholesterol or CLC (increases cholesterol) with LDL in the absence of glycerol did not yield any improvement in post-thaw motility when compared with $10 \%$ LDL controls. A previous study has shown counteraction of egg yolk effects with CLC treatments, ${ }^{20}$ thus, it is consistent with our results in the present study, in which a counteraction of LDL effects with CLC was demonstrated in a preliminary experiment, where CLC but not LDL treatment showed inferior post-thaw motility (data not shown). Reduction of the $\mathrm{C} / \mathrm{P}$ ratio by supplementing with phosphatidylcholine also did not yield any improvement in post-thaw motility when compared to $10 \%$ LDL controls. However, reducing C/P ratio by addition of MBCD (removes cholesterol) decreased post-thaw motility. These findings suggested that monkey sperm membranes in combination with LDL are already in an optimal C/P range to tolerate cryodamge. Further addition of cholesterol contributes no additional benefits, but decreasing cholesterol will have negative effects on sperm cryopreservation. The addition of LDL instead of egg yolk has been shown to increase cholesterol content of post-thaw sperm in boars. ${ }^{10}$ Therefore, the protective role of LDL in rhesus monkey sperm cryopreservation could also be due to a higher cholesterol content provided by LDL.

Based on the composition of egg yolk granules provided by Ref. 6, HDL represents about $15 \%$ of yolk dry matter. If we consider that egg yolk possesses about $50 \%$ dry matter, then the $20 \%$ egg yolk extender contains approximately $1.5 \%(\mathrm{w} / \mathrm{v})$ HDL. The present study revealed no differences in post-thaw motility between solution controls of TEST and TEST with addition of $1 \%-4 \%$ HDL in the presence or absence of glycerol. Our findings suggest that HDL plays a neutral role in sperm cryopreservation, neither detrimental nor beneficial. Earlier findings of the yolk granules could be misinterpreted by later studies as negative effects while their true statement was 'the granular fraction offered the least protection to the sperm cells'. ${ }^{1}$ The neutral effect of HDL found in the present study could due to the fact that
HDL precipitated out of solution upon freezing and thawing (our observation).

Despite that LDL provided equal cryoprotection as that of whole egg yolk for sperm of rhesus monkeys, and the possible advantages of eliminating progesterone and providing a more standardized extender with controlled composition, it is worth noting that some other components in whole egg yolk may also contribute to sperm post-thaw survival. For example, egg yolk is also rich in various antioxidants such as vitamins $\mathrm{E}$ and $\mathrm{A}$, and lutein as well as many amino acids. ${ }^{28} \mathrm{High}$ levels of reactive oxygen species have been associated with cryopreservation and were postulated to be at least partially responsible for decreased sperm function after thawing. ${ }^{29}$ Vitamin $\mathrm{E}$ is classified as the most potent non-enzymatic antioxidant, ${ }^{30}$ and is one of the major membrane protectants against reactive oxygen species and lipid peroxidation. ${ }^{31}$ More recently, many antioxidants have been used as supplements in extenders for cryopreservation of sperm with beneficial effects (e.g., Refs 32-34). Therefore, it is possible that antioxidants in egg yolk also partially contribute to sperm survival after thawing, which could also be one of the main reasons responsible for different effectiveness of egg yolk from various birds in sperm cryopreservation. ${ }^{35,36}$ The LDL extraction process may reduce or eliminate these antioxidants. As illustrated in this study, HDL did not actually cause any detrimental effect on sperm survival after thawing. Therefore, whole egg yolk may still have advantages over LDL, serving as an important ingredient in extenders for sperm cryopreservation in rhesus monkeys. Having said this, there could be an advantage in using LDL to replace egg yolk in human sperm cryopreservation, especially when thawed sperm will be used for Assisted Reproductive Technique (ART) procedures where removal of undefined animal products is preferred to avoid the potential for animal virus contamination. Alternatively, future studies could examine combinations of LDL with various antioxidants for their effectiveness in protecting sperm from cryodamage. Such an approach has recently been shown to be effective in sperm cryopreservation of $\operatorname{dogs}^{37}$ and bulls. ${ }^{38}$

In summary, our findings reveal that LDL is the main component in egg yolk that is responsible for its protective role for sperm cryopreservation in rhesus monkeys. Regardless of the presence or absence of glycerol, the protective role of LDL is similar to that of egg yolk and further, we did not observe any superiority in post-thaw survival with LDL when compared to egg yolk. Modifying the lipid composition of LDL-sperm membrane complex with the addition of cholesterol, CLC, and phosphatidylcholine also did not yield any improvements in postthaw survival; while addition of MBCD reduced post-thaw motility. HDL plays a neutral role in sperm cryopreservation of rhesus monkeys.

\section{AUTHOR CONTRIBUTIONS}

$\mathrm{QD}, \mathrm{SER}$ and $\mathrm{DH}$ performed all the experiments described in the manuscript. QD and CAV designed the studies, performed the data analysis, and wrote the manuscript.

\section{COMPETING FINANCIAL INTERESTS}

No conflict of interest interfered with the interpretation of the results and that of their applications.

\section{ACKNOWLEDGMENTS}

This work was supported in part by funding from the National Natural Science Foundation of China (No. 30800845), the Natural Science Fund for Distinguished Young Scholars of Zhejiang Province (No. R3100105), and the NIH grants RR00169 and RR13439. We thank Dr. M. Anton for providing the detailed protocols of LDL and HDL extraction. 
1 Pace MM, Graham EF. Components in egg yolk which protect bovine sperm during freezing. J Anim Sci 1974; 39: 1144-9.

2 Watson PF. Electroejaculation, semen characteristics and semen preservation of the brindled gnu. J Reprod Fert 1976; 47: 123-6.

3 Watson PF. The roles of lipid and protein in the protection of ram spermatozoa at 5 degree C by egg-yolk lipoprotein. J Reprod Fertil 1981; 62: 483-92.

4 Foulkes JA. The separation of lipoproteins from egg yolk and their effect on the motility and integrity of bovine spermatozoa. J Reprod Fertil 1977; 49: 277-84.

5 Moussa M, Martinet V, Trimeche A, Tainturier D, Anto M. Low density lipoproteins extracted from hen egg yolk by an easy method: cryoprotective effect on frozen-thawed bull semen. Theriogenology 2002; 57: 1695-1706.

6 Anton M. Composition and structure of hen egg yolk. In: Huopalahti R, Lopez-Fandino $\mathrm{R}$, Anton M, Schade R, editors. Bioactive Egg Compounds. Berlin/Heidelberg: Springer-Verlag; 2007. pp1-6.

7 Powrie WD, Nakai S. The chemistry of eggs and egg products. In: Stadelman WJ, Cotterill OJ, editors. Egg Science and Technology. Westport, , CT: Avi Publishing; 1986. pp97-139.

8 Thérien I, Moreau R, Manjunath P. Bovine seminal plasma phopholipid-binding proteins stimulate phospholipids efflux from epididymal sperm. Biol Reprod 1999; 61: 590-8

9 Bencharif D, Amirat L, Anton M, Schmitt E, Desherces S et al. The advantages of LDL (low density lipoproteins) in the cryopreservation of canine semen. Theriogenology 2008; 70: 1478-88.

10 Yamauchi S, Nakamura S, Lay KM, Azuma T, Yakabi T et al. Characteristics of Okinawan native Agu pig spermatozoa after addition of low-density lipoprotein to freezing extender. J Reprod Dev 2009; 55: 558-565.

11 Sarason RL, VandeVoort CA, Mader DR, Overstreet JW. Electroejaculation by direct penile stimulation of restrained but unanesthetized macaques. J Med Primatol 1991; 20: $122-5$.

12 Dong Q, Rodenburg SE, Huang C, VandeVoort CA. Effect of pre-freezing conditions on semen cryopreservation of rhesus monkey. Theriogenology 2008; 70: 61-9.

$13 \mathrm{Li} \mathrm{YH}$, Cai KJ, Kovacs A, Ji WZ. Effects of various extenders and permeating cryoprotectants on cryopreservation of cynomolgus monkey (Macaca fascicularis) spermatozoa. J Androl 2005; 26: 387-95.

14 Anton M. High-density lipoproteins (HDL) or lipovitellin fraction. In: Huopalahti R, Lopez-Fandino R, Anton M, Schade R, editors. Bioactive Egg Compounds. Berlin/ Heidelberg: Springer-Verlag; 2007. pp13-6.

15 Dong Q, Correa LM, VandeVoort CA. Rhesus monkey sperm cryopreservation with TEST-yolk extender in the absence of permeable cryoprotectant. Cryobiology 2009; 58: 20-7.

16 Rutllant J, Pommer AC, Meyers SA. Osmotic tolerance limits and properties of rhesus monkey (Macaca mulatta) spermatozoa. J Androl 2003; 24: 534-41.

17 Agca Y, Mullen S, Liu J, Johnson-Ward J, Gould K et al. Osmotic tolerance and membrane permeability characteristics of rhesus monkey (Macaca mulatta) spermatozoa. Cryobiology 2005; 51: 1-14.

18 Alapati R, Goff K, Kubisch MH, Devireddy RV. Water transport in epididymal and ejaculated rhesus monkey (Macaca mulatta) sperm during freezing. Cryobiology 2008; 57: 182-5

19 Martin WG, Tattrie NH, Cook WH. Lipid extraction and distribution studies of egg yolk lipoproteins. Can J Biochem Physiol 1963; 41: 657-66.

20 Purdy PH, Graham EF. Effect of cholesterol-loaded cyclodextrin on the cryosurvival of bull sperm. Cryobiology 2004; 48: 36-45.

21 Ricker JV, Linfor JJ, Delfino WJ, Kysar P, Scholtz EL et al. Equine sperm membrane phase behavior: the effect of lipid-based cryoprotectants. Biol Reprod 2006; 74: 359-65.

22 Drobnis EZ, Crowe LM, Berger T, Anchordoguy TJ, Overstreet JW et al. Cold shock damage is due to lipid phase transitions in cell membranes: a demonstration using sperm as a model. J Exp Zool 1993; 265: 432-7.

23 Parks JE, Lynch DV. Lipid composition and thermotropic phase behavior of boar, bull, stallion, and rooster sperm membranes. Cryobiology 1992; 29: 255-66.

24 Darin-Bennett A, White G, Hoskins DD. Phospholipids and phospholipid-bound fatty acids and aldehydes of spermatozoa and seminal plasma of rhesus monkeys. J Reprod Fert 1977; 49: 119-22.
25 Grunze M, Deutike B. Changes of membrane permeability due to extensive cholesterol depletion in mammalian erythrocytes. Biochim Biophys Acta 1974; 356: 125-30.

26 Zeng WX, Terada T. Effects of methyl-beta-cyclodextrin on cryosurvival of boar spermatozoa. J Androl 2001; 22: 111-8.

27 Zeron Y, Tomczak M, Crowe J, Arav A. The effect of liposomes on thermotropic membrane phase transitions of bovine spermatozoa and oocytes: implications for reducing chilling sensitivity. Cryobiology 2002; 45: 143-52.

28 Seuss-Baum I. Nutritional evaluation of egg compounds. In: Huopalahti R, LopezFandino R, Anton M, Schade R, editors. Bioactive Egg Compounds. Berlin/Heidelberg: Springer-Verlag; 2007. pp117-44.

29 Chatterjee S, Gagnon C. Production of reactive oxygen species by spermatozoa undergoing cooling, freezing and thawing. Mol Reprod Dev 2001; 59: 451-8.

30 Silva PFN. Physiology of peroxidation process in mammalian sperm. PhD thesis, Utrech University, Ridderkerk, The Netherlands, 2006. pp5-36

31 Surai P, Kostjuk I, Wishart G, Macpherson A, Speake B et al. Effect of Vitamin E and selenium supplementation of cockerel diets on glutathione peroxidase activity and lipid peroxidation susceptibility in sperm, testes and liver. Biol Trace Elem Res 1998; 64: 119-32.

32 Andrabi SM, Ansari MS, Ullah N, Afzal M. Effect of non-enzymatic antioxidants in extender on post-thaw quality of buffalo (Bubalus bubalis) bull spermatozoa. Pakistan Vet J 2008; 4: 159-62.

33 Großfeld R, Sieg B, Struckmann C, Frenzel A, Maxwell WM et al. New aspects of boar semen freezing strategies. Theriogenology 2008; 70: 1225-33.

34 Dong Q, Tollner TL, Rodenburg SE, Hill DL, VandeVoort CA. Antioxidants, Oxyrase and mitochondrial uncoupler 2, 4-dinitrophenol improved post-thaw survival of rhesus monkey sperm from ejaculates with low cryosurvival. Fertil Steril2010; 94: 2359-61.

35 Andrabi SM, Ansari MS, Ullah N, Anwar M, Mehmood A et al. Duck egg yolk in extender improves the freezability of buffalo bull spermatozoa. Anim Reprod Sci 2008; 104: 427-33.

36 Su L, Li X, Quan J, Yang S, Li Y et al. A comparison of the protective action of added egg yolks from five avian species to the cryopreservation of bull sperm. Anim Reprod Sci 2008; 104: 212-9.

37 Bencharif D, Amirat L, Pascal O, Anton M, Schmitt E et al. The advantages of combining low-density lipoproteins with glutamine for cryopreservation of canine semen. Reprod Dom Anim 2010; 45: 189-200.

38 Amirat-Briand L, Bencharif D, Vera-Munoz O, Bel Hadj Ali H, Destrumelle S et al. Effect of glutamine on post-thaw motility of bull spermatozoa after association with LDL (low density lipoproteins) extender: preliminary results. Theriogenology 2009; 71: 1209-14.

39 VarelaJrAS, Corcini CD, Ulguim RR, Alvarenga MV, Bianchi I et al. Effect of low density lipoprotein on the quality of cryopreserved dog semen. Anim Reprod Sci 2009; 115: 323-27.

$40 \mathrm{Hu}$ JH, Li QW, Zan LS, Jiang ZL, An JH et al. The cryoprotective effect of low-density lipoproteins in extenders on bull spermatozoa following freezing-thawing. Anim Reprod Sci 2010; 117: 11-7.

41 Vera-Munoz O, Amirat-Briand L, Diaz T, Vasquez L, Schmidt E et al. Effect of semen dilution to low-sperm number per dose on motility and functionality of cryopreserved bovine spermatozoa using low-density lipoproteins (LDL) extender: comparison to Trilady $\mid \circledR$ and Bioxcell囚. Theriogenology 2009; 71: 895-900.

42 Amirat L, Tainturier D, Jeanneau L, Thorin C, Gerard O et al. Bull semen in vitro fertility after cryopreservation using egg yolk LDL: a comparison with Optidyl, a commercial egg yolk extender. Theriogenology 2004; 61: 895-907.

$43 \mathrm{Hu} \mathrm{JH}$, Li QW, Jiang ZL, Li WY. Effects of different extenders on DNA integrity of boar spermatozoa following freezing-thawing. Cryobiology 2008; 57: 257-62.

44 Jiang ZL, Li QW, Hu JH, Li WY, Zhao HW et al. Improvement of the quality of boar cryopreservation semen by supplementing with low density lipoprotein in diluents. Cryobiology 2007; 54: 301-4.

45 Jiang ZL, Li QW, Li WY, Hu JH, Zhao HW et al. Effect of low density lipoprotein on DNA integrity of freezing-thawing boar sperm by neutral comet assay. Anim Reprod Sci 2007; 99: 401-7.

46 Martinez-Pastor F, Martinez F, Alvarez M, Maroto-Morales A, Garcia-Alvarez $\mathrm{O}$ et al. Cryopreservation of Iberian red deer (Cervus elaphus hispanicus) spermatozoa obtained by electroejaculation. Theriogenology 2009; 71: 628-38.

47 Hong W, Ni L, Tian D, Lu Y, Deng C et al. Effect of LDL on cryopreservation of sheep spermatozoa. Anim Husbandry Feed Sci 2008; 4: 29-31 (In Chinese with English abstract). 